\title{
A Case of Pituitary Stalk Interruption Syndrome in Early Childhood Presenting with Congenital Hypothyroidism
}

\author{
Min Kyeong Seong, So Yoon Jung, Jeongho Lee, Dong Hwan Lee \\ Department of Pediatrics, Soonchunhyang University Seoul Hospital, Soonchunhyang University College of Medicine, Seoul, Korea
}

\begin{abstract}
Pituitary stalk interruption syndrome (PSIS) manifests as an isolated or combined pituitary hormone deficiency (CPHD) and is usually diagnosed by magnetic resonance imaging (MRI). We describe a patient with PSIS who presented with central hypothyroidism. The patient was born at 35 weeks' gestation and weighed 1,980 g. Newborn screening test including thyroid-stimulating hormone (TSH) were normal; however, follow-up tests revealed low serum free thyroxine and mildly elevated TSH levels. Thyroid hormone replacement was initiated on the 29th day of birth. At 13-month-old age, the TRH stimulation test showed a delayed and prolonged increase in serum TSH levels, suggesting tertiary hypothyroidism. The combined pituitary stimulation test revealed CPHD and hyper-responsiveness of prolactin to TRH, suggesting hypothalamic-pituitary disconnection. MRI of the pituitary gland revealed the absence of the pituitary stalk, a small anterior pituitary, and an ectopic posterior pituitary located in the hypothalamic area. Replacement therapies with corticosteroid and growth hormone were initiated and then she reached catch-up growth. The TRH stimulation test can be helpful for diagnosing central hypothyroidism, which may be an early presentation of PSIS. Therefore, patients with central hypothyroidism should be considered for PSIS, thereby patients can be treated earlier and reach normal growth and development.
\end{abstract}

Keywords: Pituitary stalk interruption syndrome; Combined pituitary hormone deficiency; Hypothyroidism

\section{INTRODUCTION}

Ectopic posterior pituitary (EPP), referred to as the pituitary stalk interruption syndrome (PSIS), is characterized by an absent or thin pituitary stalk, hypoplasia of the anterior pituitary and/or an EPP [1]. It manifests as an isolated or combined pituitary hormone deficiency (CPHD), which varies in severity and onset [2]. Although the exact prevalence is unknown, a recent study reported that approximately $50 \%$ of the patients with hypopituitarism showed these findings on magnetic resonance imaging (MRI) [3]. The exact etiology of PSIS remains unclear. Perinatal events causing hypoxia are considered possible contributors, based on the high prevalence of breech delivery, cesarean section, and asphyxia in patients with PSIS [4]. However, current data support the hypothesis that describes an association between PSIS and defective embryogenesis. Mutations of the genes involved in the early devel- opment of the hypophysis (LHX4, OTX2, SOX3, and PROKR2) have been identified in patients with PSIS [5]. However, these are reported in less than $5 \%$ of the patients; therefore, the most of causative genes remain to be determined [6].

Patients with PSIS are usually referred for the evaluation of growth retardation during the first decade of life. Patients usually show isolated growth hormone (GH) deficiency when initially diagnosed; however, other pituitary hormone deficiencies leading to CPHD are identified later in approximately $50 \%$ of the patients. Neonates presenting with hypoglycemia, jaundice, and/or micropenis may often be diagnosed with PSIS. Growth retardation may not be evident during this stage of development; therefore, clinicians must remain mindful of this possibility when evaluating neonates and infants. Patients are treated with substitution of deficient hormones. Therefore, early and accurate diagnosis of hormone deficiency is important for prompt initiation of treatment [7]. 


\section{CASE REPORT}

A 13-month-old girl was referred for the re-evaluation of congenital hypothyroidism that was diagnosed shortly after birth. The patient was born via spontaneous vaginal delivery at 35 weeks of gestation and weighed 1,980 $\mathrm{g}$ (10th-50th percentile) at birth. Preterm birth was attributed to maternal preeclampsia. She showed peripheral cyanosis and a weak cry at birth with 1- and 5-minute Apgar scores of 8 and 10, respectively. She was admitted to the neonatal intensive care unit for 8 days because of prematurity and low birth weight. Initial thyroid-stimulating hormone (TSH) by newborn screening test (NST) was normal (3.5 $\mu \mathrm{IU} / \mathrm{mL}$; normal range for this age group $<12.0 \mu \mathrm{IU} / \mathrm{mL}$ ). At 15 days of age, her serum free thyroxine (T4) was low at $0.793 \mathrm{ng} / \mathrm{dL}$ (normal range for this age group, $0.93-1.70 \mathrm{ng} / \mathrm{dL}$ ), and TSH was mildly elevated at $5.86 \mu \mathrm{IU} / \mathrm{mL}$ (normal range for this age group, $0.5-5.6 \mu \mathrm{IU} / \mathrm{mL}$ ). Follow-up tests on the 29th day of birth revealed that serum free T4 is constantly low at $0.824 \mathrm{ng} / \mathrm{dL}$ and TSH was not elevated (5.37 $\mu \mathrm{IU} / \mathrm{mL}$ ). She was diagnosed with hypothyroidism based on low free T4, and thyroid hormone replacement therapy was initiated for normal development.

She was $74 \mathrm{~cm}$ tall (10th-25th percentile), weighed $8.86 \mathrm{~kg}$ (10th25th percentile), and showed normal development milestones for her 13 months of age. Physical and neurological examinations were unremarkable. She took levothyroxine $20 \mu$ g once daily and the recent thyroid function test was normal. We reviewed her medical records and suspected central hypothyroidism because initial compensatory TSH elevation was absent despite low free T4. The thyrotropin-releasing hormone $(\mathrm{TRH})$ stimulation test was planned to diagnose central hypothyroidism. After discontinuing levothyroxine for 2 weeks, laboratory investigations showed a low serum free T4 level of $0.53 \mathrm{ng} / \mathrm{dL}$ (reference range, $0.8-2.2 \mathrm{ng} / \mathrm{dL}$ ), low triiodothyronine of $92.53 \mathrm{ng} / \mathrm{dL}$ (reference range, 119-218 ng/dL),
TSH of $1.69 \mu \mathrm{IU} / \mathrm{mL}$ (reference range, $0.5-4.8 \mu \mathrm{IU} / \mathrm{mL}$ ). The TRH stimulation test was performed; serum TSH levels were measured at baseline and 30, 60, 90, and 120 minutes after intravenous administration of $7 \mathrm{mg} / \mathrm{kg}$ of TRH. We observed a delayed and prolonged increase in serum TSH levels (Fig. 1). Thyroid ultrasonography and scan revealed no abnormalities in the size, shape, and location of the thyroid gland. It suggested central hypothyroidism, especially tertiary hypothyroidism, and we took further tests for the assessment of other hormone deficiencies. The combined pituitary stimulation test was performed with intravenous administration of $0.1 \mathrm{unit} / \mathrm{kg}$ of regular insulin and $0.1 \mathrm{mg}$ of gonadotropin-releasing hormone. There was no proper cortisol response to the insulin tolerance test. The peak GH was low at $1.67 \mathrm{ng} / \mathrm{mL}$ and peak luteinizing hormone $(\mathrm{LH})$ and follicle-stimulating hormone (FSH) levels were elevated for her age at $10.93 \mathrm{mIU} / \mathrm{mL}$ and 24.94 $\mathrm{mIU} / \mathrm{mL}$, respectively. This test revealed CPHD (GH and LH/FSH deficiency) and impaired hypothalamic-pituitary-adrenal axis in addition to hyper-responsiveness of prolactin (PRL) to TRH, suggesting hypothalamic-pituitary disconnection (i.e., loss of the normal dopaminergic inhibition of PRL secretion) (Table 1). MRI of the pituitary gland revealed an absence of the pituitary stalk, a

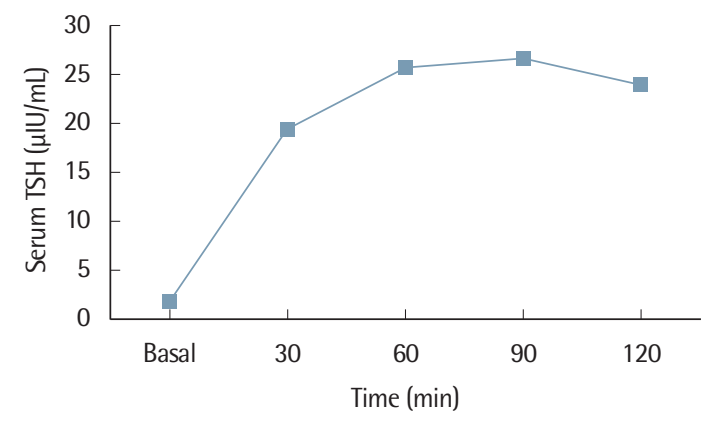

Fig. 1. TRH stimulation test showed a delayed and prolonged increase in serum TSH levels. TRH, thyrotropin-releasing hormone; TSH, thyroid-stimulating hormone.

Table 1. Combined pituitary function test (after intravenous administration of $7 \mathrm{mg} / \mathrm{kg}$ of TRH, $0.1 \mathrm{unit} / \mathrm{kg}$ of Rl and $0.1 \mathrm{mg}$ of GnRH) results performed at 13 -monthold age

\begin{tabular}{lcccccccc}
\hline Variable & Glucose $(\mathrm{mg} / \mathrm{dL})$ & $\mathrm{PRL}(\mathrm{ng} / \mathrm{mL})$ & $\mathrm{TSH}(\mu \mathrm{lU} / \mathrm{mL})$ & $\mathrm{LH}(\mathrm{mlU} / \mathrm{mL})$ & $\mathrm{FSH}(\mathrm{mlU} / \mathrm{mL})$ & $\mathrm{GH}(\mu \mathrm{g} / \mathrm{L})$ & Cortisol $(\mu \mathrm{g} / \mathrm{dL})$ & $\mathrm{ACTH}(\mathrm{pg} / \mathrm{mL})$ \\
\hline Reference range & - & $3-24$ & $0.5-4.8$ & $0.02-1.8$ & $1.0-4.2$ & $0.7-6$ & $3-21$ & $10-60$ \\
Basal & 82 & 1.62 & 1.69 & 2.56 & 8.35 & 0.52 & 3.13 & 13.64 \\
$30 \mathrm{~min}$ & 42 & 33.10 & 19.48 & 10.93 & 17.58 & 0.35 & 6.94 & 14.95 \\
$60 \mathrm{~min}$ & 69 & 31.17 & 25.77 & 10.52 & 21.41 & 0.67 & 5.11 & 11.38 \\
$90 \mathrm{~min}$ & 82 & 32.09 & 26.65 & 10.74 & 20.45 & 1.55 & 3.73 & 9.98 \\
$120 \mathrm{~min}$ & 81 & 30.06 & 24.0 & 9.41 & 24.94 & 1.67 & 3.63 & 10.91 \\
\hline
\end{tabular}

TRH, thyrotropin-releasing hormone; Rl, regular insulin; GnRH, gonadotropin-releasing hormone; PRL, prolactin; TSH, thyroid-stimulating hormone; LH, luteinizing hormone; FSH, follicle stimulating hormone; $\mathrm{GH}$, growth hormone; $\mathrm{ACTH}$, adrenocorticotropic hormone. 
Seong MK, et al. • PSIS Presenting with Congenital Hypothyroidism

small anterior pituitary, and an EPP located in the hypothalamic area (Fig. 2A, B). There were no mutations related to PSIS in genetic analysis using Whole Exome Sequencing including LHX4, OTX2,

\section{SOX3, and PROKR2.}

The patient continued to receive thyroid hormone supplementation and corticosteroid replacement was added. When she was
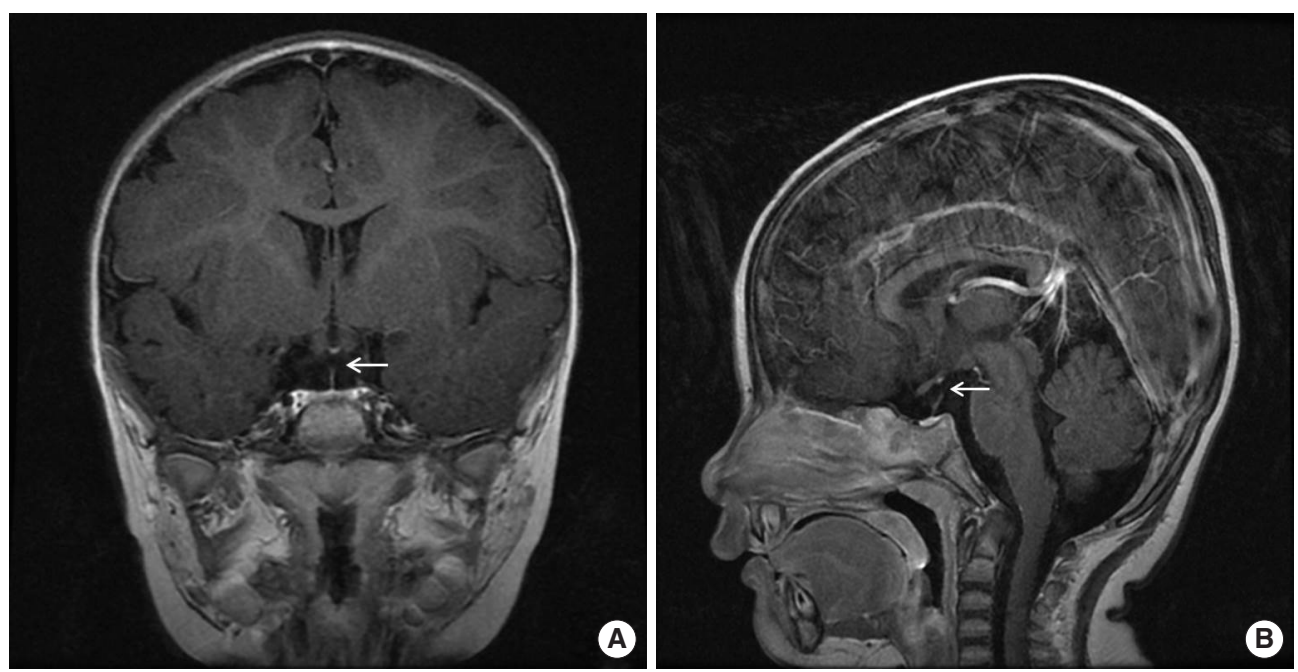

Fig. 2. Brain magnetic resonance imaging showing $T 1$ coronal view $(A)$ and $T 1$ sagittal view (B). The pituitary gland is small in size (approximately $2.5 \mathrm{~mm}$ ) with a flattened appearance. The neurohypophysis is not well visualized at its normal location, and a small mild T1 high signal intensity enhancing focus, suspicious for ectopic neurohypophysis, is observed in the hypothalamic area. The upper portion of the pituitary stalk is not well delineated (arrow).
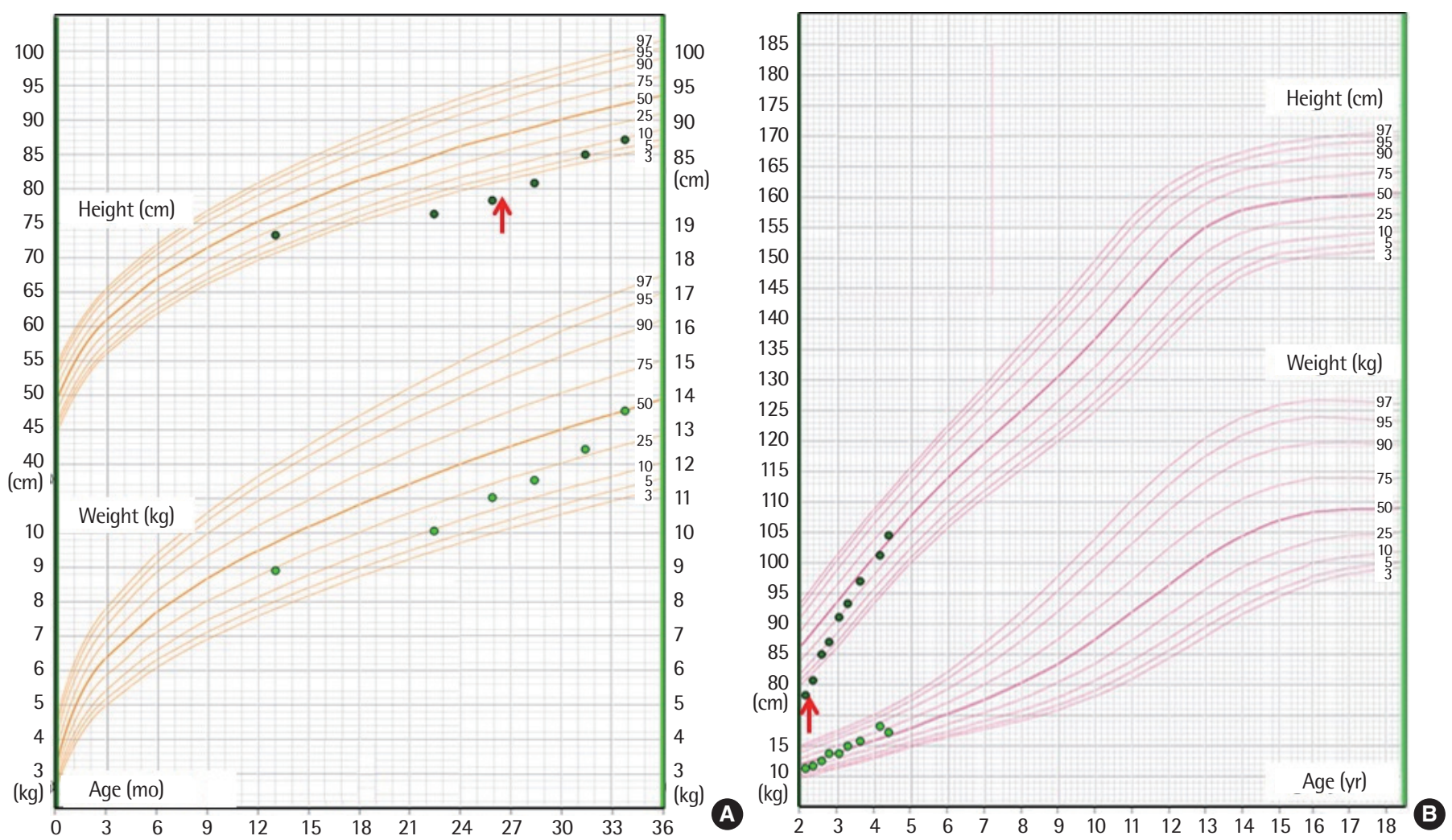

Fig. 3. Growth chart of the patient. (A) Her height became below the 3rd percentile at 21-month-old age compared with normal height at 13-month-old age. Her growth velocity slowed down. (B) After initiating growth hormone replacement (arrow), catch-up growth was observed. 
26-month-old age, she became short stature $(78 \mathrm{~cm}$ tall; below the 3 rd percentile) as her growth velocity slowed down and weighed $11 \mathrm{~kg}$ (10th-25th percentile); therefore, GH replacement therapy was initiated $(0.31 \mathrm{mg} / \mathrm{kg} / \mathrm{wk})$. Catch-up growth was observed during the first year of treatment (growth velocity $8-10 \mathrm{~cm} / \mathrm{yr}$ ), and her height was $101.1 \mathrm{~cm}$ (50th percentile) at 4 years of age (Fig. 3). She had no clinical evidence of central diabetes insipidus and developmental delay.

\section{DISCUSSION}

The patient was suspected as central hypothyroidism by the results of low free T4 without compensatory TSH elevation. By performing the TRH stimulation test, she was diagnosed as tertiary hypothyroidism and undergone the combined pituitary stimulation test. It revealed CPHD and hypothalamic-pituitary disconnection. She was confirmed as PSIS by the findings of MRI. She is in normal development with receiving thyroid hormone, corticosteroid and GH replacement therapy, and sex hormone replacement is also planned for her pubertal age.

Recent guidelines for the management of congenital hypothyroidism recommend treatment initiation in patients with belownormal levels of free T4 or elevated TSH levels exceeding $20 \mu \mathrm{IU} /$ $\mathrm{mL}$ [8]. Central hypothyroidism is often diagnostically challenging owing to the variable clinical presentation and laboratory findings. Patients usually present with low serum free T4 levels; however, TSH levels may be markedly low, normal, or even slightly elevated. Additionally, some patients with central hypothyroidism have normal TSH with low-normal serum free T4. Most centers check only TSH levels in neonates, which can delay the diagnosis of not only central hypothyroidism but also a combined hormonal disorder [9].

The TRH stimulation test is a simple and safe diagnostic tool for the evaluation of central hypothyroidism and was previously routinely used as a component of the diagnostic work-up in patients with central hypothyroidism. However, owing to its low sensitivity, this test has shown limited applicability and is deemed unsuitable for routine testing after the introduction of sensitive TSH assays. Atmaca et al. [10] investigated the usefulness of the TRH stimulation test in patients with central hypothyroidism. The TRH stimulation test was conducted in 72 patients with Sheehan's syndrome; 10 patients (13.8\%) with normal and low-normal serum free $\mathrm{T} 4$ showed a delayed or prolonged response to the TRH stimulation test. Based on these results, the authors concluded that the TRH stimulation test is useful to diagnose central hypothyroidism, particularly in patients with low-normal serum free T4 and/or TSH levels and known hypothalamic-pituitary pathology.

In the majority of patients, congenital hypothryodism occurs in combination with additional pituitary hormone deficits. Coexisting ACTH or GH deficiency may cause significant risks like lifethreatening hypoglycemia. Hence, evaluation of the hypothalamic-pituitary axis should be conducted in patients with congenital hypothyroidism. MRI is also a gold standard imaging modality to the assess hypothalamic-pituitary axis. Its results can guide genetic testing, and both MRI and genetic analysis may predict and alert clinicians toward the evolution of other hormone deficits [11].

The patient underwent further thyroid function test because she was a preterm baby. If she had taken only routine NST, which includes just TSH level, the diagnosis of hypothyroidism would have been late. Also, she was diagnosed with central hypothyroidism and CPHD, which can affect the growth and development of a child, based on the findings of the TRH stimulation test. Therefore, simultaneous free T4 and TSH measurements are recommended in neonates with perinatal events. Central hypothyroidism should be considered when the patient has low serum free T4 with normal or mildly increased TSH levels. And, the TRH stimulation test is a useful diagnostic aid in such cases.

Early and accurate diagnosis of hormone deficiency is important in initiating prompt treatment. Due to the current NST protocol and the various clinical presentation and laboratory findings of central hypothyroidism, the diagnosis would be delayed. The TRH stimulation test could be helpful in differentiating central hypothyroidism, which may be an early presentation of PSIS. Therefore, patients with central hypothyroidism should be considered for PSIS, thereby patients can be treated earlier and reach normal growth and development.

\section{REFERENCES}

1. Fujisawa I, Kikuchi K, Nishimura K, Togashi K, Itoh K, Noma S, et al. Transection of the pituitary stalk: development of an ectopic posterior lobe assessed with MR imaging. Radiology 1987;165:487-9.

2. Pinto G, Netchine I, Sobrier ML, Brunelle F, Souberbielle JC, Brauner R. Pituitary stalk interruption syndrome: a clinical-biological-genetic assessment of its pathogenesis. J Clin Endocrinol Metab 1997;82:3450-4.

3. Deal C, Hasselmann C, Pfaffle RW, Zimmermann AG, Quigley CA, Child CJ, et al. Associations between pituitary imaging abnormalities and clinical and biochemical phenotypes in children with congenital growth hormone deficiency: data from an international observational study. 
Horm Res Paediatr 2013;79:283-92.

4. Kikuchi K, Fujisawa I, Momoi T, Yamanaka C, Kaji M, Nakano Y, et al. Hypothalamic-pituitary function in growth hormone-deficient patients with pituitary stalk transection. J Clin Endocrinol Metab 1988;67:817-23.

5. Tajima T, Ishizu K, Nakamura A. Molecular and clinical findings in patients with LHX4 and OTX2 mutations. Clin Pediatr Endocrinol 2013; 22:15-23.

6. Karaca E, Buyukkaya R, Pehlivan D, Charng WL, Yaykasli KO, Bayram Y, et al. Whole-exome sequencing identifies homozygous GPR161 mutation in a family with pituitary stalk interruption syndrome. J Clin Endocrinol Metab 2015;100:E140-7.

7. Bar C, Zadro C, Diene G, Oliver I, Pienkowski C, Jouret B, et al. Pituitary stalk interruption syndrome from infancy to adulthood: clinical, hormon- al, and radiological assessment according to the initial presentation. PLoS One 2015;10:e0142354.

8. Leger J, Olivieri A, Donaldson M, Torresani T, Krude H, van Vliet G, et al. European Society for Paediatric Endocrinology consensus guidelines on screening, diagnosis, and management of congenital hypothyroidism. J Clin Endocrinol Metab 2014;99:363-84.

9. Gupta V, Lee M. Central hypothyroidism. Indian J Endocrinol Metab 2011;15(Suppl 2):S99-106.

10. Atmaca H, Tanriverdi F, Gokce C, Unluhizarci K, Kelestimur F. Do we still need the TRH stimulation test? Thyroid 2007;17:529-33.

11. Schoenmakers N, Alatzoglou KS, Chatterjee VK, Dattani MT. Recent advances in central congenital hypothyroidism. J Endocrinol 2015;227: R51-71. 